\title{
A PÓS-GRADUAÇÃO EM GEOGRAFIA NO BRASIL: UMA CONTRIBUIÇÃO À POLÍTICA DE AVALIAÇÃO
}

José Borzacchiello da Silva Prof. da Universidade Federal do Ceará borza@ufc.br

Eustógio Wanderley Correia Dantas Prof. da Universidade Federal do Ceará edantas@ufc.br

\section{Resumo}

No momento em que o processo de avaliação da pósgraduação em geografia suscita discussões e críticas, a presente pesquisa adquire caráter provocativo. Resulta de levantamento de dados e informações do sistema CAPES/ $\mathrm{CNPq}$, gerando possibilidade de cruzamento da produção científica docente do conjunto dos programas e cursos de pós-graduação em geografia. Certamente contribuirá para o aprimoramento do processo de avaliação em curso, apontando a necessidade de reflexão constante, evitando enfoque produtivista e fragmentado, que gera competição entre os programas e cursos.

Palavras-chave: Pós-graduação em geografia, avaliação, produtividade.

\begin{abstract}
As the process of assessing postgraduate work in geography has raised discussions and criticism, this research has a challenging character. It comes from data collecting and information of CAPES/CNPq system, opening possibilities for crossing teaching scientific production of the programmes and courses of postgraduate work in geography. It will certainly contribute to improving the current evaluating process, pointing out the need for constant reflection, avoiding productivist, fragmented focus, which creates competition among programmes and courses.
\end{abstract}

Key Words: Postgraduate work in Geography, evaluation, productivity.

\section{$\circ \bigcirc 0$}

\section{Introdução}

As expectativas frente a realização do VI Encontro Nacional da ANPEGE em Fortaleza, no período de 28 a 30 de setembro, motivou a elaboração da presente pesquisa, abrindo espaço para uma discussão necessária e pertinente. Além do mais, a publicação do n. ${ }^{\circ} 2$ da Revista da ANPEGE, aumentou o entusiasmo para que a pesquisa se consolidasse. Muito já foi dito e discutido e muito pouco escrito sobre a trajetória da pós-graduação em geografia no Brasil. Entretanto, malgrado o interesse, quando se busca dados e informações consistentes, capazes de balizar parâmetros e escolhas, constata-se o quanto tem que ser feito na perspectiva de se oferecer à comunidade científica um quadro mais completo a respeito da pós-graduação em geografia no país.

Partimos da constatação que a opacidade em relação a alguns programas e cursos, decorre da falta de pesquisas e mesmo da dificuldade de divulgação daquelas existentes. Nosso pressuposto básico parte da afirmativa de que o conhecimento do panorama geral da pós-graduação no país está calcado no senso comum, e de per si, mapeia a distribuição dos cursos, contemplando, aqueles programas há muito conso- 
lidados que contam em seus corpos docentes, com professores de renome nacional e internacional. A pós-graduação em geografia no Brasil, em seus primórdios, dependia das universidades estrangeiras para formação de seus quadros. ${ }^{1}$ É considerável o número de professores que obtiveram seus títulos de doutorado no exterior, principalmente na França. ${ }^{2}$ Inicialmente, apoiou-se no tripé São Paulo, Rio de Janeiro e Recife. O destaque adquirido por esses cursos garantiu ampla visibilidade aos seus professores-orientadores, ao mesmo tempo, em que favoreceu o desenho de enormes áreas de influência no território nacional.

Os cursos de pós-graduação em geografia da Universidade de São Paulo (Geografia Física e Geografia Humana) são pioneiros. Criados em 1971, exerceram papel preponderante no processo de formação de quadros propiciando a criação de novos cursos. A centralidade exercida pelos cursos pioneiros findou estabelecendo amplo intercâmbio entre as poucas instituições de ensino superior brasileiras, com os recém criados cursos de São Paulo. A UFRJ implantou seu curso em 1972. A afirmação dos cursos de graduação nas capitais dos estados e, posteriormente, nas principais cidades do interior, exerceu forte compressão para criação de novos cursos de pós-graduação. Essa demanda apresentou resultados positivos. Em 1996 contávamos com 11 cursos de pós-graduação em funcionamento. (Tabela 1).

Tabela 1 - Cursos de Pós-Graduação em Funcionamento: 1996, 2001 e 2004

\begin{tabular}{|c|c|c|c|}
\hline 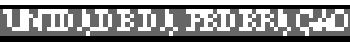 & pHT & mi & mitis \\
\hline \multicolumn{4}{|l|}{ 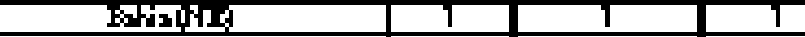 } \\
\hline 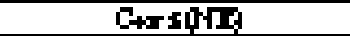 & - & 1 & 2 \\
\hline 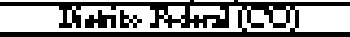 & E & $T$ & $T$ \\
\hline \multicolumn{4}{|l|}{ 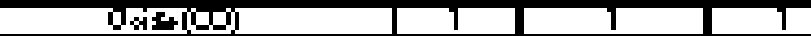 } \\
\hline 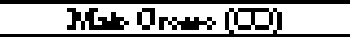 & - & - & 1 \\
\hline 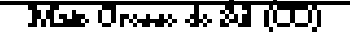 & - & - & $T$ \\
\hline 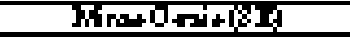 & 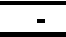 & 3 & 3 \\
\hline hrsitㅁ] & $=$ & - & 1 \\
\hline Jatbitrit & - & - & 1 \\
\hline jerst & - & 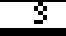 & 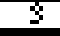 \\
\hline \multicolumn{4}{|l|}{ Jerandes t'Lt } \\
\hline $\mathrm{F}+\mathrm{d}+\mathrm{T}$ rirsRI & 1 & 3 & 3 \\
\hline 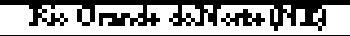 & - & $T$ & $\mathbf{T}$ \\
\hline 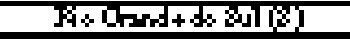 & - & $T$ & 1 \\
\hline \multicolumn{4}{|l|}{ 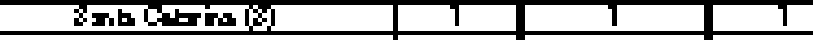 } \\
\hline $25 \mathrm{H}+\mathrm{B}$ & d & 4 & 5 \\
\hline $2+m+4+1$ & 1 & 1 & 1 \\
\hline & 11 & $z$ & $\boldsymbol{D}$ \\
\hline
\end{tabular}

Fonte: CAPES ANPEGE

O progressivo aumento da demanda pela pós-graduação em geografia alcança maior respaldo quando ocorre a conquista de licença de afastamento para realização de cursos de pós-graduação, com a conseqüente liberação de professores de suas cargas de aula e pesquisa junto aos Departamentos. Atrela-se a este fato a política de concessão de bolsas de estudos por parte das agências de fomento. Essas e outras iniciativas exerceram uma aceleração no processo de criação de novos cursos. Em 2001, conforme dados obtidos junto a CAPES e ANPEGE, eram 23 os cursos de pós-graduação em funcionamento (Tabela 1). 
Cabe mencionar que, independente do avanço político e da visibilidade obtida pela pós-graduação, eram muitas as dificuldades encontradas pelas universidades para implantar seus cursos de pós-graduação. Independente da demanda, a carência de professores qualificados, portadores da titulação exigida pelas agências de fomento e órgãos superiores das Instituições de Ensino Superior, era um forte entrave.

Para ultrapassar esses limites e barreiras, vários cursos recorriam ao expediente de solicitar aos docentes mais titulados, disponibilização de seus currículos visando a inclusão de seus nomes nos processos endereçados aos órgãos competentes para serem autorizados e posteriormente, reconhecidos. Na medida em que os Departamentos formavam seus quadros, ampliava o número de cursos de pós-graduação em geografia.

A pós-graduação em nossa área continuou apresentando uma curva tendencial ascendente, expressando forte dinâmica quando em 1993 foi criada a ANPEGE (Associação Nacional de Pós-graduação e Pesquisa em Geografia). Os antecedentes de sua criação remontam à uma década. Preocupados em dar corpo às reivindicações dos cursos de pós-graduação, profissionais ligados aos cinco primeiros Programas, criados a partir de 1971, em reunião realizada em 1984, esboçaram as bases do que viria a ser a ANPEGE. Tratava-se do I Encontro Nacional de Pós-Graduação em Geografia. ${ }^{3}$

A pós-graduação alcançou considerável nível de abrangência no território nacional, o que lhe permite excepcional cobertura. Entretanto, o quadro ainda é bastante promissor, considerando que na região Norte do país, só foi instalado o recém criado curso de Belém, na UFPA. No Nordeste, São Luiz, Terezina e Maceió, para falar apenas das capitais, não contam ainda com a pós-graduação stricto sensu. No Sudeste, Vitória do Espírito Santo é a única capital sem um curso de pós-graduação em geografia.

O processo de instalação de novos cursos intensificou-se nos últimos anos. Em 2004 eram 29 os cursos de mestrado e 14 os de doutorado, funcionando nos 29 programas e cursos (Tabela 1). O processo mantem seu dinamismo ao ponto de ter sido aprovado recentemente (2005) pelo CTC CAPES: o doutorado da UFPR, em Curitiba, e três mestrados (PUC/MG/SP, UNIR, UEPG).

Esses programas e cursos, são periodicamente avaliados, produzindo um ranking da pós-graduação no país. A propósito da avaliação, qualquer ação deve considerar o fator tempo. São muitos os cursos recémcriados que seguem à risca os procedimentos impostos pelas agências de fomento e controle. Falar de controle é falar de avaliação. Os critérios adotados pela CAPES tem sido objeto de contínuas discussões, algumas acaloradas, chegando, muitas vezes a extremos - os que aceitam e defendem plenamente e os que fazem severas críticas. Independente do reconhecimento da necessidade da avaliação são comuns os rumores de um caráter produtivista, por estabelecer uma "guerra" entre os programas e cursos rumo a conceitos mais altos. Uma das primeiras críticas foi feita por Queiroz Neto(1984), ao afirmar:

um exemplo curioso deste fenômeno é a importância crescente dos aspectos burocráticos, representativos entre outros pela imensa e inútil papelada que tem que produzir anualmente sob a forma de programas e relatórios a serem enviados à CAPES, que mudou suas diretrizes e passa a exercer o papel de órgão normalizador.

Numa escala que varia de 1 a 7, os cursos de pós-graduação em geografia sujeitos à avaliação institucional apresentaram os seguintes resultados na última avaliação trienal (2001-2003) (Tabela 2).

Tabela 2: Avaliação Trienal da Pós-Graduação em Geografia 


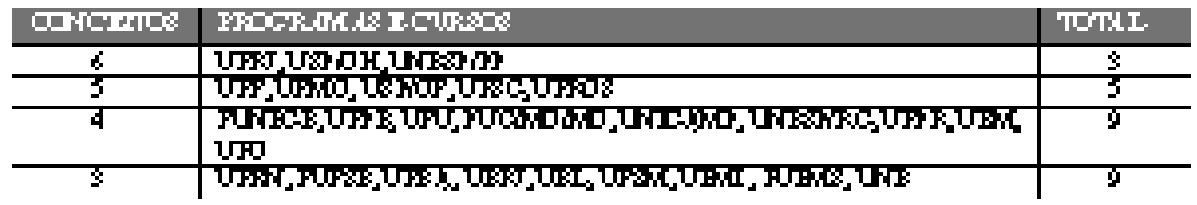

Fonte: CAPES

\footnotetext{
Nota: os cursos implantados em 2004 não foram avaliados, considerando, na presente pesquisa, os conceitos atribuídos em suas criações pelo CTC CAPES.
}

Observa-se a predominância do Sudeste quantos aos programas que obtiveram os conceitos mais elevados. Cabe ressaltar que essa região conta com dez (10) dos cursos de doutorado do total de quatorze (14) existentes. Os demais se localizam no Nordeste (2), e no Sul (2).

\section{A produção docente: carro chefe da avaliação?}

Com tradição de quase trinta anos de avaliação da pós-graduação no Brasil, a CAPES obtém reconhecimento nacional e internacional, chegando ao ponto de empreender reformas, na década de 1990, nos termos das empreendidas nos "países centrais" (Moreira; Hortale; Hartz , 2004). Somado a esta experiência, no momento atual, a comunidade acadêmica é chamada a contribuir no aprimoramento do processo de avaliação a partir de discussões empreendidas entre coordenações de programas, cursos e seus comitês de área.

Tal abertura nos anima no sentido de contribuir no aprimoramento do processo de avaliação implementado, com análise da produção docente nos últimos três anos: 2002, 2003 e 2004. A premissa seria a de percebermos, no cerne de nossa área, a necessidade de implementação de uma política de avaliação que possibilite estabelecer comparações entre os diversos programas e cursos, numa perspectiva horizontal e não tão somente vertical, focada em cursos isolados que geram, conseqüentemente, dados fragmentados.

No nosso entender, a contribuição dos programas e cursos, na definição de critérios de avaliação, pode ser potencializada com o conhecimento detalhado das características marcantes de seus pares e do conjunto que compõe os programas ou cursos, condição "sine qua non" ao estabelecimento de metas e diretrizes, pautadas nas experiências e práticas daqueles que compõem e constroem a pós-graduação.

A análise detalhada, refinada e seqüenciada dos programas e cursos de forma verticalizada nos deixa dependentes e suscetíveis de diretrizes externas que, em última instância, podem distanciar-se do perfil real e possível dos programas e cursos aos quais nos vinculamos.

É conveniente a passagem de uma avaliação pautada nos programas e cursos para uma outra de caráter mais amplo, capaz de externar o perfil da área. Nestes termos, nos dispomos, a partir da consideração da produção docente em Geografia, indicar quadro definidor do perfil da área em foco, podendo com isto estabelecer discussões e trocas com outras áreas e, principalmente, com os programas e cursos que vivenciam a pós-graduação.

A disponibilização de dados pela CAPES e CNPq facilita nossa tarefa, podendo suscitar quadro analítico 
possibilitador do atendimento de nosso intento. Pautados em sistema de informação recentemente disponibilizado por estas instituições de fomento, procedemos montagem de quadros, tabelas e gráficos representativos da produção docente, especificamente livros (autoria/organizados), capítulos de livros e artigos publicados em periódicos.

Acessando ao sistema supracitado, disponibilizado em http://ged.capes.gov.br/AgProd/silverstream/ pages/pgRelBolsistasProdPesq.html, coletou-se dados vinculados às Unidades da Federação, Instituições de Ensino Superior, Áreas de Avaliação (no caso em Geografia), Programas e Docentes (NRD0 a NRD6), em seguida procedeu-se primeira coleta de dados em setembro de 2004, sendo realizada atualização e conferência em maio de 2005.

Dos 29 programas e cursos criados até 2004, o sistema de informação CAPES/CNPq somente disponibilizou dados dos 26 já consolidados, não abarcando os novos cursos: UFPb, UFC e UFPA, iniciados em 2004. No sentido de não se limitar apenas à análise parcial dos dados dos programas e cursos existentes, optou-se por consulta direta a professores integrantes dos três cursos em questão, para a obtenção de listagem de seus respectivos corpos docentes. Em seguida a pesquisa foi completada nos mesmos termos das demais, com consulta direta à Plataforma Lattes do CNPq.

A sistematização dos dados obtidos gerou quadro inicial com: a) indicação dos programas e cursos por estado/região e, nestes, das instituições de ensino possuidoras de programas (m/d - mestrado e doutorado) ou cursos (m-aqueles que contam somente com o mestrado) de pós-graduação stricto sensu com seus conceitos (3 a 6); b) número de docentes por Instituição de Ensino Superior (IES); c) quantitativo absoluto e relativo da produção de livros, capítulos de livros e artigos em periódicos; d) cômputo total da produção dos anos 2002 a 2004; e) média da produção (cômputo total/pelo número de anos em evidência, 3). (Quadro 1).

\section{Tentativa de interpretação e análise dos dados}

$\mathrm{Na}$ Área de Geografia, no período analisado, foram publicados 494 livros (autoria/organizado), 1.189 capítulos de livro e 2.067 artigos em periódicos (Quadro 1).

Considerando a distribuição da produção por região, nota-se presença de programas e cursos nas regiões Sudeste (10 programas, 5 em São Paulo, 3 em Minas Gerais e 2 no Rio de Janeiro; 1 curso no Rio de Janeiro); Nordeste ( 2 programas, 1 em Pernambuco e 1 em Sergipe; 5 cursos, 2 no Ceará, 1 na Bahia, 1 no Rio Grande do Norte e 1 na Paraíba); Sul (2 programa em Santa Catarina e Rio Grande do Sul; 4 cursos, 3 no Paraná e 1 no Rio Grande do Sul); Centro Oeste (4 cursos, 1 no Distrito Federal, 1 em Goiás, 1 em Mato Grosso e 1 em Mato Grosso do Sul); Norte (1 curso no Pará) (Figura 1). 


\begin{tabular}{|c|c|c|c|c|c|c|c|c|}
\hline \multirow{2}{*}{ 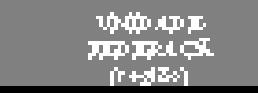 } & \multirow{2}{*}{ 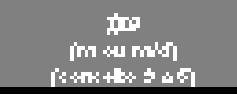 } & \multirow{2}{*}{ 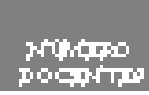 } & \multicolumn{2}{|c|}{ 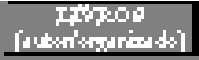 } & \multicolumn{2}{|c|}{ 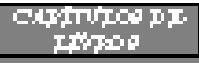 } & \multicolumn{2}{|c|}{ 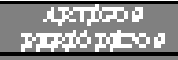 } \\
\hline & & & abteluts & ralios & bestuk & rithios & kashes & raves \\
\hline Estidpors & Yrakimibi & 15 & 18 & $1 *$ & so & $\mathbf{s} \boldsymbol{\alpha}$ & ᄀ & 856 \\
\hline \multirow{2}{*}{ Ounphy } & $y+m_{1}(\theta)$ & 11 & $s$ & $s$ & 34 & 590 & w & $s$ \\
\hline & $r$ respimile & 16 & 纤 & 15 & so & 170 & $\underline{x}$ & וג5 \\
\hline Figik 7 derd 100 ) & utsimin & 11 & 1 & 0,8 & 10 & $\theta$ & 116 & $10 \% 4$ \\
\hline asidupe) & Yroimilen & 15 & 11 & 1,8 & 58 & sy & 4 & $5 \$ 1$ \\
\hline $\mathrm{rab}$ O $\mathrm{m}=1 \mathrm{CO}$ & 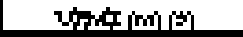 & 14 & $t$ & $0 ; 7$ & 16 & 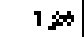 & 4 & 534 \\
\hline 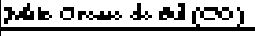 & Indesimis) & 16 & $1 s$ & 1,8 & As & 13 & $\pi$ & $5 \% 4$ \\
\hline \multirow{3}{*}{$\operatorname{ris}$} & Yhoomsing & 16 & 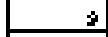 & os & $\Sigma 6$ & 1 & $s$ & ונג \\
\hline & 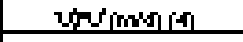 & 17 & 7 & OA & $s_{2}$ & 1 的 & 105 & 605 \\
\hline & 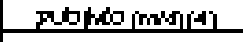 & 15 & 15 & s & 68 & $4 x$ & $\pi$ & $5 \%$ \\
\hline Fund ph & Uromimin & 11 & 7 & ogs & 56 & $s, 5$ & 호도 & 200 \\
\hline Tumbars or & บrseimin & 14 & 1 & $0>0$ & $\$ 1$ & 15 & $s s$ & 570 \\
\hline \multirow{3}{*}{ Darurd (O) } & Leximis & 厸 & 16 & $0 / 2$ & 45 & $1 \%$ & 16 & $6 \%$ \\
\hline & 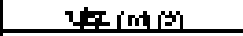 & 16 & 11 & ogs & 46 & $\$ \$ 7$ & $\omega$ & $5 \% 5$ \\
\hline & 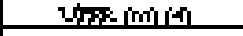 & 17 & $\leq$ & $0 \%$ & 26 & $1 \geqslant$ & $\pi$ & 276 \\
\hline 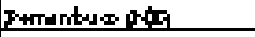 & Yrreimulien & 10 & 11 & נו & 15 & 15 & $\underline{x}$ & 58 \\
\hline \multirow{3}{*}{ Pis dotarins (PST) } & Uretimsion & $=4$ & 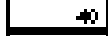 & דגו & $\$ 5$ & $\Delta>s$ & 童 & $2 \mathrm{~A} 1$ \\
\hline & 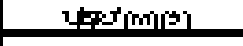 & 16 & 17 & 100 & 18 & 130 & $s$ & SAds \\
\hline & Urimbigis) & 15 & $\approx$ & $1 \%$ & $s-1$ & 4)s & $\underline{x}$ & $\$ 76$ \\
\hline Fis Orumd+dorstst+pos & Yroximisel & 18 & 14 & 18 & 15 & $1 \%$ & 호 & 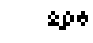 \\
\hline \multirow{2}{*}{ xis orumd +d od (U) } & usorimisis & 18 & 의 & נו & so & 1 A & 1 些 & SAN \\
\hline & Yreodimulas & 16 & 15 & ops & 54 & $\mathbf{s} \mathbf{3}$ & s & 575 \\
\hline Art Ourire (O) & Y reo (mulis) & $\infty$ & 14 & 0 & 18 & os & $\approx$ & $s$ \\
\hline \multirow{5}{*}{ 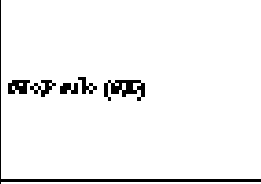 } & 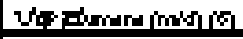 & $s$ & 15 & נוs & 114 & $s$ & $\%$ & 1 \\
\hline & 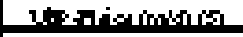 & $\mathbf{s}$ & 1 & oper & $\$ 1$ & 124 & 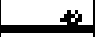 & \&ss \\
\hline & 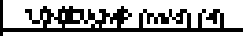 & 17 & 18 & 07 & 56 & $\mathbf{s}>\mathrm{s}$ & $\pi$ & 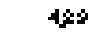 \\
\hline & 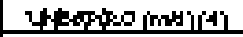 & $\omega$ & $=4$ & 8 & $g \hat{z}$ & 85 & $1 \%$ & $5 \% 8$ \\
\hline & 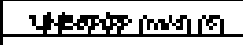 & $s 0$ & 고 & $1 \%$ & $\Delta s$ & 45 & 167 & 285 \\
\hline atriftos & rysimbivin & 17 & $\Delta$ & $1 \% \mathrm{~s}$ & 51 & $s$ & 110 & $6 A 7$ \\
\hline \multirow{3}{*}{\multicolumn{2}{|c|}{ 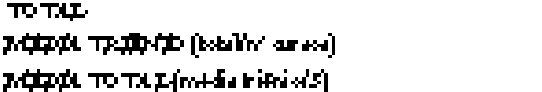 }} & sת & $\$$ & sopo & $116 \%$ & $\operatorname{ars}$ & 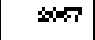 & $117 \$ 6$ \\
\hline & & & $17 \mathrm{as}$ & $0 \%$ & 11 & 28 & 7187 & 406 \\
\hline & & & $s h$ & 0,55 & 1586 & $o>4$ & 257 & 135 \\
\hline
\end{tabular}

Fonte: Sistema de Informação CAPES-CNPq; Currículo Lattes CNPq

Organizado Por: Eustógio Dantas e José Borzacchiello da Silva

Figura 1: Número de Programas e Cursos Por Região/Unidade da Federação 


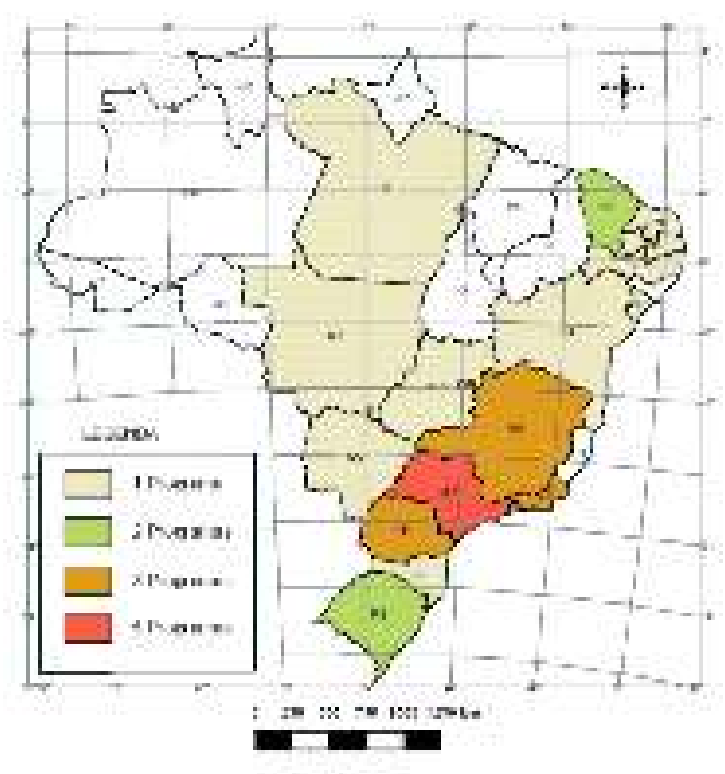

Fonte CAPES, 2004

Além da concentração geográfica dos programas e cursos no Sudeste $(11 / 29$ - 37,92\%), seguido do Nordeste (7/29 - 24,14\%), Sul (6/29 - 20,69\%), Centro Oeste (4/29 - 13,79\%) e, por último, o Norte (1/29 - 3,46\%), dá-se, também, a concentração da produção. Dos livros, $47,37 \%$ da produção é atinente ao Sudeste, seguido em ordem decrescente pelo Nordeste, Sul, Centro Oeste e Norte, com os respectivos valores: $27,33 \%, 16,59 \%, 7,29 \%$ e 1,42\%. No tópico capítulos de livros, a concentração é mais acentuada, sendo o Sudeste responsável por 54,33\%, seguido pelo Nordeste 18,84\%, o Sul 16,48\%, o Centro Oeste 7,15\% e o Norte 3,2\%. Quanto aos artigos publicados, o Sudeste concentra 41,21\%, vindo na seqüência o Sul, Nordeste, Centro Oeste e Norte, com, respectivamente, 27,34\%, 17,13\%, 13,2\% e $1,12 \%$ (Gráfico 1). 


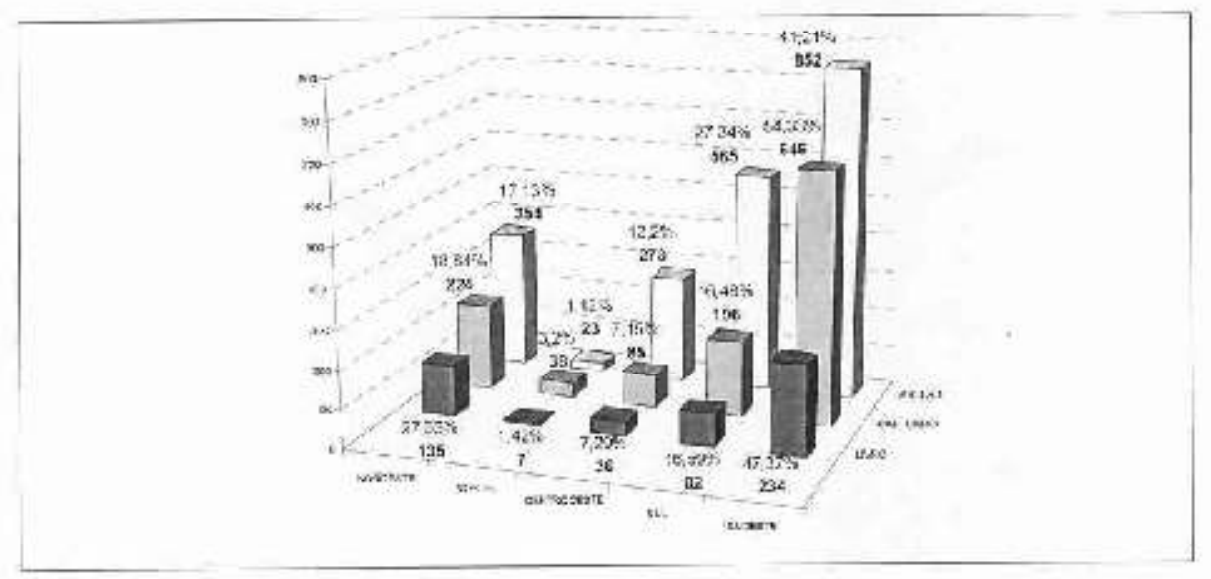

Organizads Per: Eu, fug o Taztase Jose Bazzochielio da Silcs

Embora se evidencie política da CAPES no sentido de ampliar a pós-graduação em geografia, ela não é seguida, pautando-nos nos dados absolutos aqui indicados, de uma aproximação dos percentuais de produção. Grosso modo, podemos vincular tal comportamento ao perfil dos 9 programas instalados no Sudeste. Para se ter uma idéia, considerando-se: a) o conceito máximo obtido (6) na última avaliação (triênio 2001-2003), tem-se uma razão da ordem de 3/3, ou seja, a concentração de 100\% naquela região; b) o contingente dos mesmos, no tocante aos demais programas, tem-se uma razão e de 9/13 (69,24\% dos programas situam-se também no Sudeste).

Para proceder análise mais acurada optamos por dimensionar os dados em escala menor, por programa ou curso, e em três níveis de produtividade (nível I, menor incidência de publicações; nível II, incidência média; nível III, maior incidência) para efeito de comparação da produção docente nos tópicos livros, capítulos de livros e artigos.

No caso da produção de:

. LIVROS, as freqüências atribuídas foram de: a) 0 a 14 unidades, com 15 instituições envolvidas - USPFÍSICA, UNB, UFPB, UFPR, UFU, UFPA, UFMT, UFMG, UFPE, UFG, UEL, UNICAMP, UFMS, UFSC e UFRN, com 51,73\% dos 29 cursos existentes; b) 15 a 28, abarcando 9 instituições - UFRGS, PUC/MG-MG, UEM, UERJ, UFBA, UFSM, UFF, FUFSE e FUNECE,com 31,03\% dos cursos; c) 29 a 43, com 5 instituições - UNESP/PP, UFC, UNESP/RC, UFRJ e USP-HUMANA, com 17,24\% dos cursos (Gráfico 2);

. CAPÍTULOS DE LIVROS, as freqüências adotadas foram as de: a) 0 a 28 unidades, com 12 instituições incluídas - UNB, UFPE, UFRN, UFMT, UFSC, UERJ, UFPB, USP-Física, UFMS, UFPR, UFMG e UFSM, com 41,37\% dos cursos existentes; b) 29 a 57, com 12 instituições inscritas - UFG, UFU, UFC, UFRGS, UFBA, UFPA, UNICAMP, UEM, UEL, FUNECE, FUFSE e UFF, com 41,37\% dos cursos; c) 58 a 114, com participação de 5 instituições - PUC/MG/MG, UNESP/RC, UNESP/PP, UFRJ, e USP- 
. ARTIGOS, as freqüências consideradas foram: a) 0 a 48 unidades, na qual se inserem 12 instituições - UFPA, UFRN, UFBA, UFPE, UFF, UFMG, UERJ, UFG, UFC, UFMT, UFPR e PUC/MG, com 41,37\% dos cursos existentes; b) 49 a 97, contando com 10 instituições - USP-Física, UFPB, FUNECE, UEL, USP-Humana, UFMS, UNICAMP, UFRJ, UFRGS e UFSC, com 34,49\% dos cursos; c) 98 a 145, com participação de 7 instituições - UFU, FUFSE, UNB, UFSM, UNESP/RC, UEM e UNESP/PP, com $24,14 \%$ dos cursos (Gráfico 4).

A análise comparativa dos gráficos, elaborados a partir dos dados de produção do Quadro 1 e consoante os três níveis de produtividade, com os conceitos obtidos pelos programas e cursos no triênio 2001-2003 (conforme http://capes.gov.br/capes/portal/conteudo/AvTrienal2004 FinalPorArea.pdf) conduziu à seguinte constatação:

1. Dos três programas conceito 6 (UFRJ, UNESP/PP e USP-Humana), somente a UNESP se insere exclusivamente no nível III, estando a UFRJ e USP-Humana inseridas, no tópico artigos no nível II e nos demais no nível III;

2. Dos cinco programas conceito 5 (UFMG, UFF, UFRGS, UFSC e USP-Física) percebe-se não participação deles no nível III. Encontram-se distribuídos entre os níveis I e II, sendo o mais estável a UFRGS, constante em todos os tópicos de produção no nível II e o menos estável a UFMG, sempre presente no nível I. A UFSC e a USP-Física possuem comportamentos semelhantes, estando ambas no tópico artigos no nível II e nos dois demais no nível I. A UFF, nos tópicos livros e capítulos de livros situa-se no nível II e no tópico artigo no nível I;

3. Dos programas (PUC/MG/MG, UFU, UFPE, UNESP/RC e UNICAMP) e cursos (FUNECE, UFG, UEM e UFPR) conceito 4, merece destaque a UNESP/RC por constar em todos os tópicos de produção no nível III. A FUNECE é o mais estável, constando sempre no nível II. O programa da UFPE e o curso da UFPR inserem-se exclusivamente no nível I. Os demais gravitam entre o nível I, II e III, com destaque para os programas da UFU e PUC/MG, inscritos o primeiro no tópico artigos e o segundo no tópico capítulos de livros no último nível;

4. Daqueles que receberam conceito 3, dentre eles um programa (UFSE) e os cursos da UFBA, UNB, UFSM, UFMT, UEL, UERJ, UFRN e UFSM, bem como os cursos novos (UFPB, UFC e UFBA), que a critério de nosso comitê iniciam com nota 3, percebe-se comportamento seguinte: o programa da UFSE gravita do nível II ao nível III, especificamente no quesito artigos; os cursos da UFSM, UNB e UFC gravitam do nível I ao III; os cursos da UERJ, UEL, UFMS, UFBA, UFPB e UFPA do nível I ao II; os cursos da UFRN e da UFMT ficam restritos, em todos os tópicos, ao nível I. 


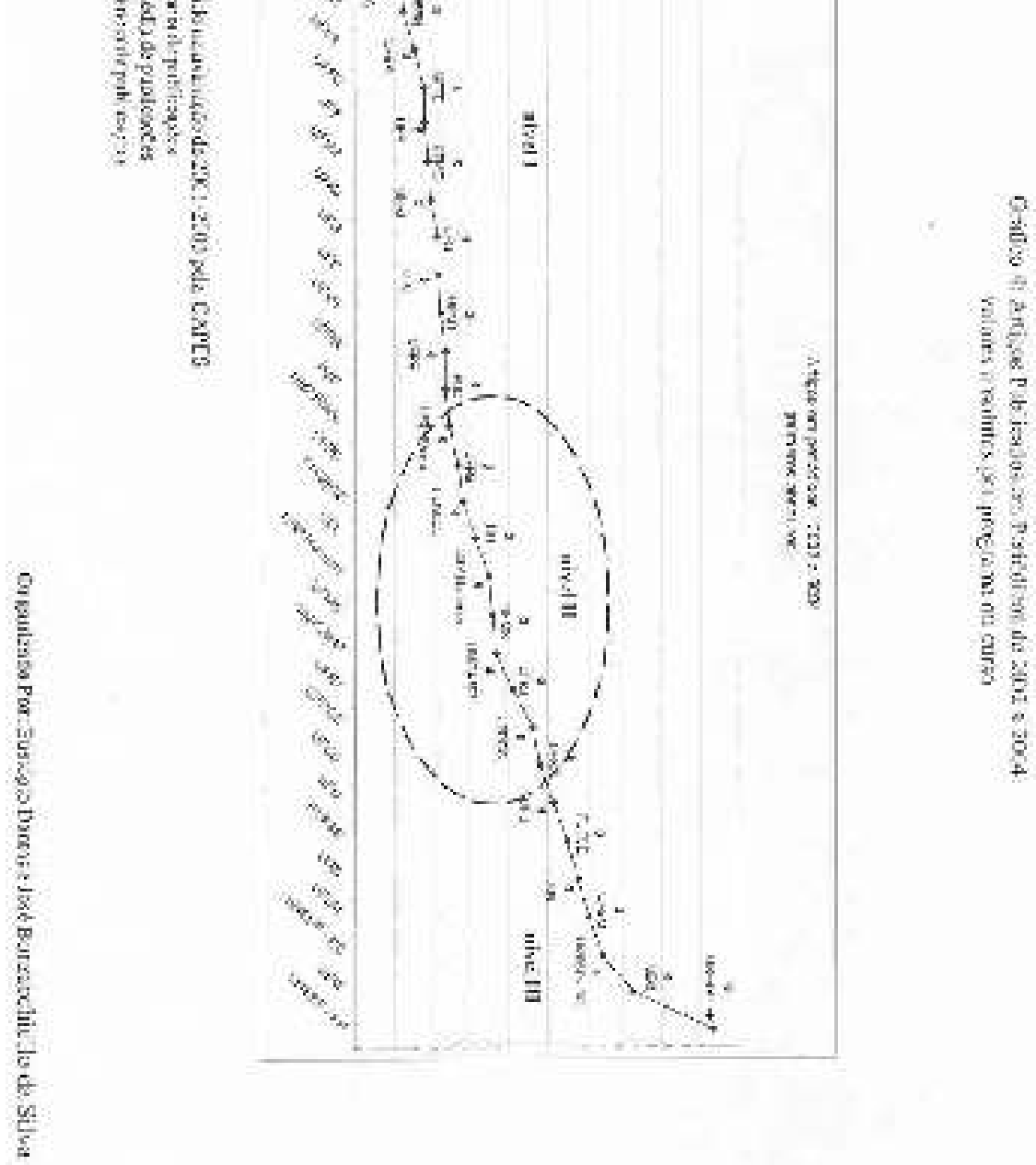

Da análise acima, com ênfase nos casos que tien ais chamaram nossa atenção, constatamos simetrias e assimetrias em relação aos conceitos atribuídos ẳos programas e cursos.

As simetrias são mais evidentes no caso dos: a) programas conceito 6, UNESP/PP, USP-Humana e UFRJ, ao gravitarem predominantemente no nível III; b) cursos conceito 5, especificamente UFRGS, estável em todos os tópicos no nível II; c) cursos conceito 4, representado pela FUNECE, participando exclusivamente do nível II.

As assimetrias evidenciam-se nos: a) programas conceito 5, na UFMG, constante em todos os tópicos de produção no nível I; b) nos programas e cursos conceito 4, com destaque para a UNESP/RC, sempre inserida no nível III e a UFU e PUC/MG que se incluem em dados tópicos no nível III, com ressalvas para a UFPE e a UFPR inscritas exclusivamente no nível I; c) programa e cursos conceito 3 , com ênfase na FUFSE ao variar do nível II ao III e ao trio UFSM, UNB e UFC, constantes, em alguns tópicos, no nível III.

Na segunda parte de nosso trabalho, se busca verticalizar ainda mais a análise ao adentrar no campo dos números relativos, na vinculação direta da produção docente com o volume do corpo docente de 31 cada um dos programas e cursos de Pós-graduação em geografia. 
A exemplo do levantamento pautado no campo do absoluto, que apresenta simetrias e assimetrias em relação aos conceitos obtidos na última avaliação da CAPES, os resultados relativos obtidos não apresentaram rebatimento com os conceitos alcançados. 


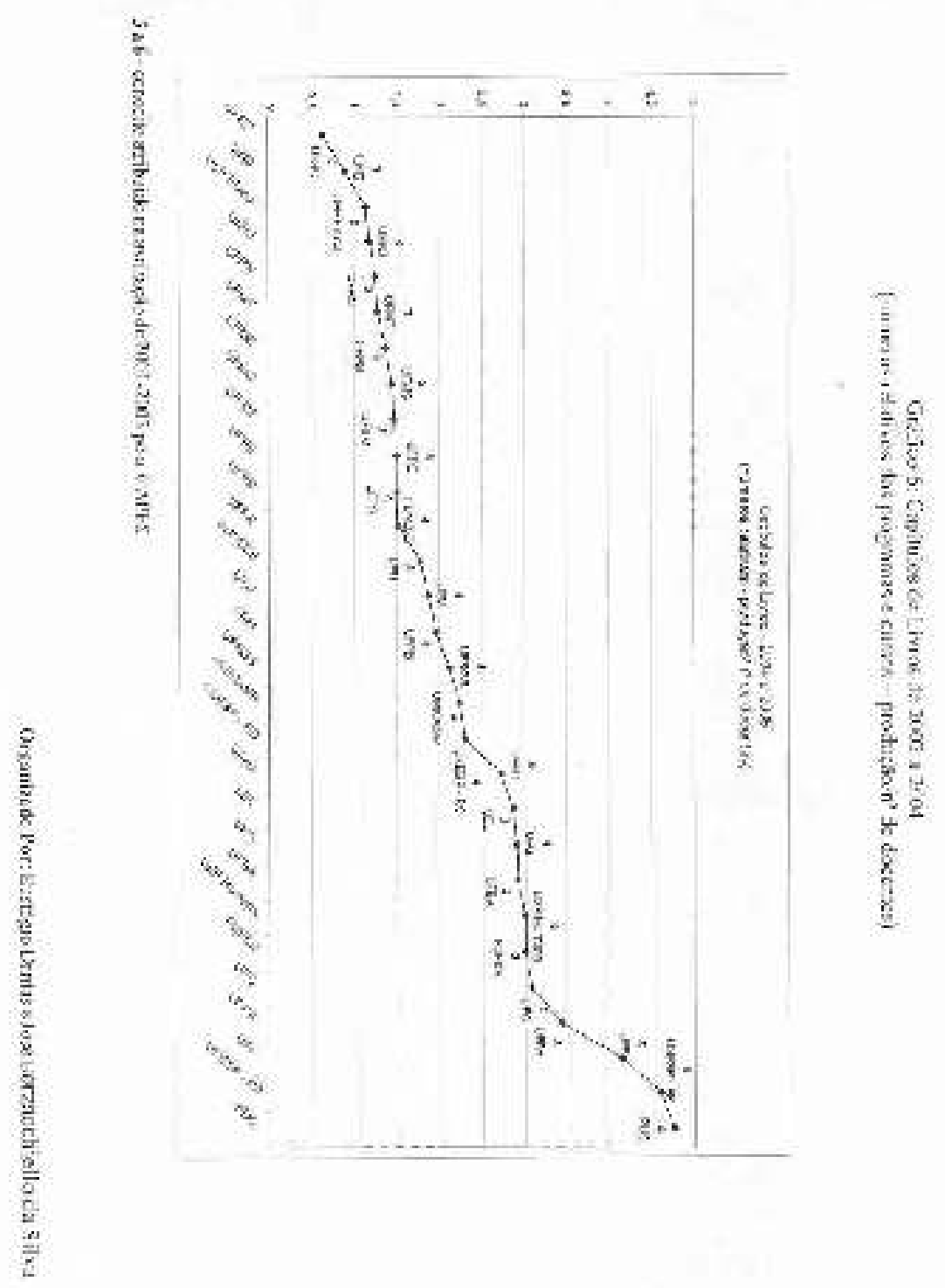




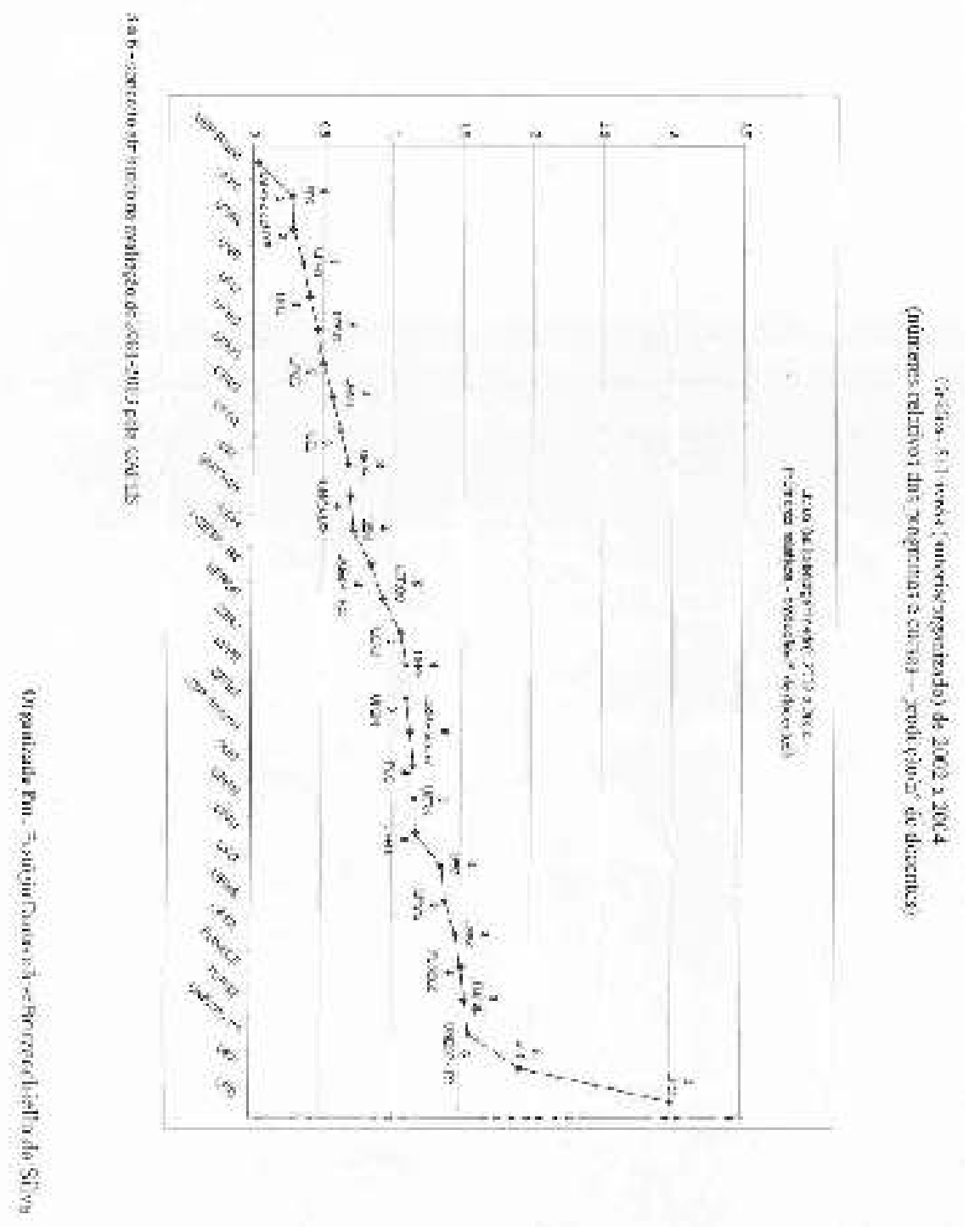




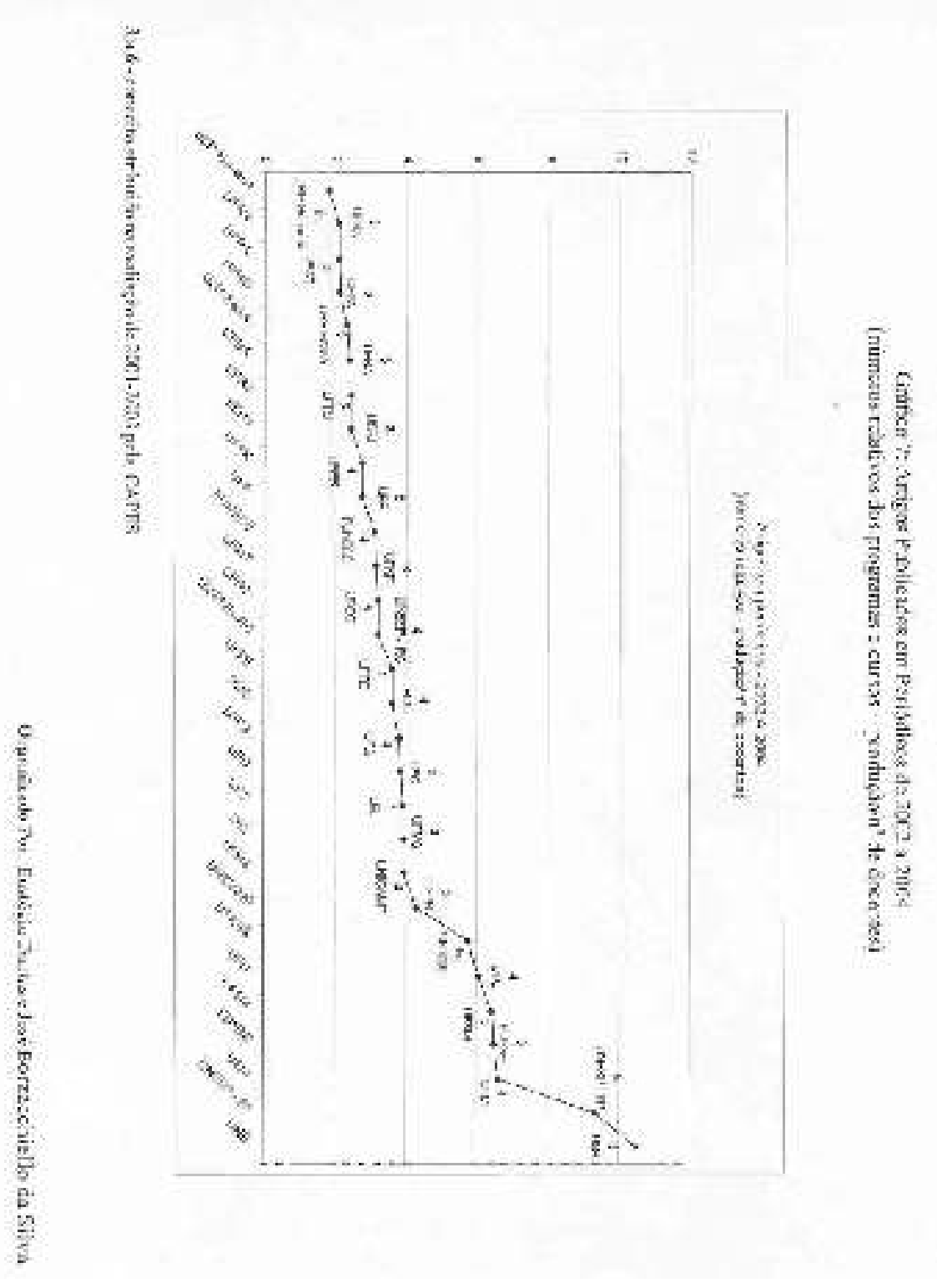

\section{Considerações Finais}

Do "compte rendu" dos dados absolutos e relativos da produção docente na pós-graduação em geografia, podemos considerar que, embora existam coincidências, torna-se impraticável adotar postura reducionista reinante e crédula de ser o conceito dos cursos diretamente relacionado à produção docente em seu estado bruto, sendo os demais quesitos meramente ilustrativos.

Do campo do absoluto percebe-se claramente, tanto simetrias como assimetrias no tocante aos conceitos obtidos, dado reforçado ao enfocar-se, no campo do relativo, a taxa individual de produtividade dos 
a) adequação dos tipos de produção à proposta do programa e vínculo com as áreas de concentração, linhas e projetos de pesquisa ou teses e dissertações;

b) qualidade dos veículos ou meios de divulgação;

c) quantidade e regularidade em relação à dimensão do NRD6; distribuição da autoria entre os docentes;

d) autoria e co-autoria de discente;

e) publicação de resultados de pesquisa não restrita aos veículos de programa.

O desdobramento da pesquisa induz à necessidade constante de se avaliar o caráter qualitativo da produção científica dos corpos docentes envolvido na pós-graduação, refreando do ímpeto produtivista..

A pesquisa apresentou resultados que exigem uma interpretação detalhada e impõem desafios. Evidencia estarem os procedimentos e critérios de avaliação cada vez mais voltados para o caráter qualitativo. Nesta direção, no processo de avaliação 2001/2003, em estreita relação com demais áreas da Grande Área de Ciências Humanas, a Comissão de Geografia teve a preocupação "de não apenas atualizar o Qualis de Periódicos da área, como também de elaborar um Qualis de Livros e um Qualis de Eventos”. (CAPES, agosto 2004).

Acreditamos ser a maior contribuição da presente pesquisa a de constatar que as leituras e avaliações elaboradas a partir de critérios puramente quantitativos tendem a induzir ao erro, principalmente se os diretamente envolvidos nela não têm conhecimento prévio do perfil daqueles que fazem a pósgraduação de sua área. $\mathrm{O}$ esforço aqui empreendido funda-se na necessidade de, no encadeamento do processo de avaliação, elaborar documentos analíticos dos resultados obtidos pelo conjunto de programas e cursos. Neste sentido foram realizados, pelos representantes das grandes áreas, estudos apontando para o desvendamentodo perfil dos profissionais que lhes caracteriza (GONDIM, 1992).

A difícil tarefa de avaliar exige postura de respeito e maturidade por parte do avaliador. Os programas e cursos não podem e não devem estabelecer suas linhas de pesquisa e planos de trabalho, preocupados apenas com o processo avaliativo que se impôs como necessidade. Na medida em que houver maior identidade e afinidade (leia-se conhecimento mútuo) entre os programas e cursos de pós-graduação em geografia e, em cada um deles, do corpo docente e discente com as linhas de pesquisa da área de concentração, a produção tenderá a adquirir um ritmo próprio, chegando a uma qualidade e, por que não dizer, originalidade de pesquisas que facilitarão o enquadramento dos programas e cursos em posições tidas como satisfatórias.

\section{Notas}

(1) O mesmo se deu com a instalação dos primeiros cursos de graduação em geografia. Foi preponderante o papel exercido por Pierre Deffontaines nas criações dos cursos da Universidade de São Paulo, em 1934, e da Universidade do Distrito Federal, em 1936.

(2) A Realização do Congresso Internacional de Geografia, da UGI (União Geográfica Internacional), realizado em 1956, no Rio de Janeiro, provocou um amplo espaço de trocas e debates que foram aprofundados com programas de intercâmbio entre França e Brasil.

(3) Anais do I Encontro Nacional de Pós-graduação em geografia, São Paulo, FAPESP/CNPq, 1984.

\section{Referências}

Anais do I Encontro Nacional de Pós-graduação em geografia, São Paulo, FAPESP/CNPq, 1984. 
CARLOS, Ana Fani Alessandri. Questões para a construção de uma política de pós-graduação em geografia, Revista da ANPEGE, nº1, 2003. p.71-83

CAPES, Avaliação da Pós-Graduação, DOCUMENTO DE ÁREA, Geografia, agosto de 2004.

GONDIM, Linda M. P. A política de fomento à pesquisa urbana: subsídios para uma avaliação das avaliações do CNPq, Revista Brasileira de Estudos Urbanos e Regionais, vol. IV, nº 1-2, 2002. p 25-36.

MOREIRA, Carlos Otávio F. Avaliação da pós-graduação: buscando consenso. RBPG. Revista Brasileira de PósGraduação, n. ${ }^{\circ} 1$ (julho), 2004 p.26-40.

QUEIRÓZ NETO, José Pereira. Pós-Graduação no Brasil: Implantação, Crescimento e Crise, Anais do I Encontro Nacional de Pós-Graduação em Geografia, São Paulo, PAPESP/CNPq, 1984.

CAPES - http://capes.gov.br/capes/portal/conteudo/AvTrienal2004_FinalPorArea.pdf

CAPES-CNPq, SISTEMA DE INFORMAÇÃO - http://ged.capes.gov.br/AgProd/silverstream/pages/pgRelBolsistasProdPesq.html

Recebido em julho de 2005

Aceito em agosto de 2005 